\title{
Onychotillomania: A Chameleon-Like Disorder: Case Report and Review of Literature
}

\author{
Polytimi Sidiropoulou $^{\mathrm{a}}$ Dimitrios Sgouros ${ }^{\mathrm{a}}$ Konstantinos Theodoropoulos ${ }^{\mathrm{b}}$ \\ Alexander Katoulis $^{b}$ Dimitrios Rigopoulos ${ }^{a}$ \\ ${ }^{a}$ 1st Department of Dermatology-Venereology, National Kapodistrian University of Athens, Medical School, \\ "A. Sygros" Hospital, Athens, Greece; ${ }^{b}$ 2nd Department of Dermatology-Venereology, National Kapodistrian \\ University of Athens, Medical School, ATTIKON University Hospital, Athens, Greece
}

\section{Established Facts}

- Onychotillomania represents a behavioral pattern characterized by repetitive self-induced trauma of the nail apparatus.

- It is categorized among obsessive-compulsive psychiatric disorders.

- The wide clinical presentations can masquerade several inflammatory skin diseases.

\section{Novel Insights}

- The current case emphasizes the chameleon-like diversity in clinical manifestations of onychotillomania.

- Onychotillomania presenting with anonychia and periungual ulceration can be an imitator of severe dermatoses such as erosive lichen planus or even nail metastasis.

- Awareness of its protean phenotype is essential for accurate diagnosis optimizing collaborative care between dermatologists and psychiatrists.

\section{Keywords}

Nails · Onychotillomania · Psychodermatology

\begin{abstract}
Onychotillomania, or nail-picking disorder, is an uncommon and misdiagnosed behavioral pattern focused on the nail apparatus. It is demarcated by the compulsive or irresistible urge in patients to constantly injure their own nails, with the fingers or tools, inflicting noticeable or even irreversible selfdestruction of the nail unit. Despite its rarity, this self-injurious coercion often poses a diagnostic and therapeutic challenge. Not only do many patients deny nail manipulation,
\end{abstract}

but also the disorder has long been recognized to present itself with a wide range of clinical features, which hampers early and indisputable diagnosis. Furthermore, onychotillomania constitutes a persistent and hardly manageable problem, mostly because of its psychocutaneous nature as well as its high propensity to coexist with underlying neuropsychiatric illnesses or other behavioral disorders. However, the medical literature concerning obsessive nail picking still remains relatively scarce. Herein, we present an extraordinary, yet very intriguing case of a Caucasian patient with onychotillomania and onychophagia coexistence masquerading a weird inflammation-like lesion.

(c) 2018 S. Karger AG, Basel

\section{KARGER}

(c) 2018 S. Karger AG, Basel

E-Mail karger@karger.com

www.karger.com/sad
Polytimi Sidiropoulou

1st Department of Dermatology-Venereology, National Kapodistrian University of Athens, Medical School, “A. Sygros” Hospital for Cutaneous and Venereal Diseases 5 I. Dragoumi street, GR-16121 Kaisariani, Athens (Greece)

E-Mail sidiropouloupolytimi@gmail.com 


\section{Introduction}

Onychotillomania represents an unusual behavioral disorder affecting the nail apparatus. It is specifically delineated by recurrent and overwhelming picking and manicuring of the nail unit, with the fingers or tools, resulting in notable onychodystrophy and, in severe cases, onychoatrophy. It may also be complicated by infections and/or paronychia [1-3]. This nail picking pattern has been classified among the field of psychocutaneous ailments, owing to its high predisposition to concomitantly manifest with neuropsychiatric comorbidities or other behavioral disorders [4-7]. According to the International Classification of Diseases-10 (ICD-10), onychotillomania falls in the spectrum of body-focused repetitive behavior disorders (BFRBDs) under the subset of other specified obsessive-compulsive and related disorders, thus overlapping between the realms of dermatology and psychiatry.

Diagnosing nail behavioral disorders can be greatly challenging not only on the grounds that patients typically deny their self-destructive compulsion, but also by virtue of the unspecific, highly variable and bizarre presentations as well as the nonspecific nature of histopathologic findings. Taken together, all these parameters may entail underrecognition, misdiagnosis, and inadequate therapeutic approach of onychotillomania, hence highlighting the pivotal role of clinicopathological confrontation towards establishment of a solid differentiation and diagnosis $[2,3]$.

Although benign nail picking is common in the general population [8], a small fraction of patients experience significant disability due to the habitual disorder $[2,6]$. Despite the incremental scientific interest in the concept of BFRBDs, onychotillomania has not been explicitly studied in respect to clinicopathologic features, associated comorbidities, and effective treatment modalities. We thereby describe an interesting case of concomitant onychotillomania and onychophagia imitating a peculiar inflammation-like lesion and we underscore that this behavioral pattern can evolve into a significant diagnostic problem in daily clinical practice.

\section{Case Report}

A 53-year-old diabetic Caucasian male presented to our tertiary referral center with a 6-month history of an inflammatory lesion on the lefthand 5th digit expanding beyond the nail unit. On physical examination, the affected skin demonstrated an inflammatory aspect, i.e., erythema, edema, and ulcerations. In addition

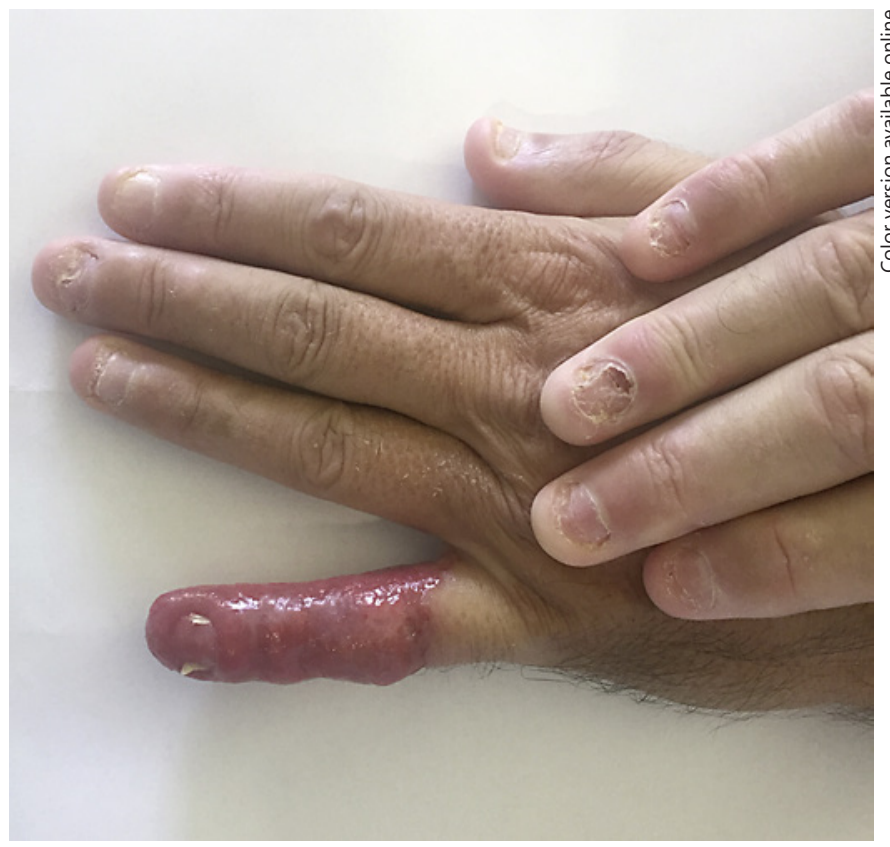

Fig. 1. Erythema, edema, and ulcerations on the left-hand 5th digit skin with anonychia and honey-colored crusts on the exposed nail bed. The nail plates of several other digits are plucked off.

to the cutaneous lesions, anonychia was also obvious, with the nail bed almost completely exposed and partially covered with yellowish, honey-colored scales and crusts (Fig. 1). No pruritus, pain, or other symptoms were reported. Many of the other fingernails were pronouncedly crumbled, splintered, and very short, with the nail bed largely exposed. The toenails were not intact. There were no features suggestive of a primary dermatosis on other areas of the skin, hair, or mucous membranes. The general and systemic examination was found to be unremarkable, while the laboratory findings were as follows: The bacterial culture of a lesion specimen revealed golden-colored colonies compatible with $S$. aureus infection. The blood tests, including WBC and CRP, were all within normal limits, whilst X-ray imaging of the digit yielded negative results.

Remarkably, the patient was initially unable to make an explanation for this condition. He was treated with combinations of oral and topical antibiotics, while an occlusive dressing bandaging the digit was daily applied. Due to rapid and significant improvement (Fig. 2), the digit bandage was withdrawn, but the patient underwent an unexpected relapse. This furnished evidence of self-inflicted behavior and led on to a further detailed interview which revealed more information pertaining to his psychiatric background. The patient finally admitted to chronically picking and chewing of his digit that was difficult if not impossible to control voluntarily. In light of these findings, the diagnosis of onychotillomania along with onychophagia was established. The psychiatric consultation sought to evaluate this clearly self-abusive demeanor unmasked an underlying depressive disorder according to the ICD-10 diagnostic criteria. 


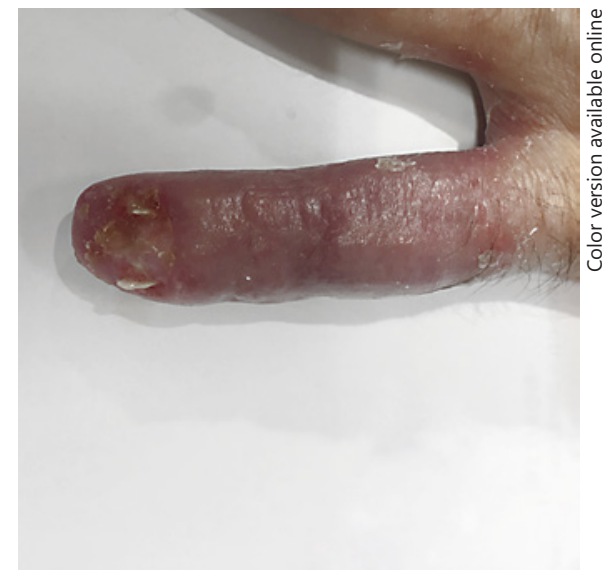

Fig. 2. Physical examination after placing the occlusive dressing shows remarkable improvement in the inflammatory aspect of the lesion.

\section{Discussion}

Onychotillomania is an unusual type of BFRBD characterized by a chronic and recurrent self-mutilating behavior directed to the nail apparatus. It is particularly defined by an irresistible urge or impulse in patients to either pick or pull at their own fingernails and/or toenails. This habitual disorder may lead to profound shortening, distortion, and, in severe cases, complete extraction of the nail plate as well as onychoatrophy, due to permanent damage of the nail matrix. The majority of nail pickers use their own fingers/nails to manipulate other digits, albeit sharp instruments, from scissors to knives or toothpicks, can be employed for excessive grooming [1-3].

Diagnosis of onychotillomania can be a real challenge for even competent skin experts. Akin to many psychodermatological disorders, clinicopathologic features of the affected nails are nonspecific, miscellaneous, and possibly bizarre. To further complicate matters, both periungual and digit cutaneous tissues may be involved. This protean phenotype renders onychotillomania a dermatological masquerader able to mimic, both clinically and histologically, various nail disease entities and may comprise a major obstacle to differential diagnostic procedure for both dermatologists and dermatopathologists [2, 3]. All these evidences prompted Reese et al. [3] in 2013 to maintain that "onychotillomania could be considered a unique variant of lichen simplex chronicus/prurigo nodularis situated at the nail unit." In our case, likewise, as a result of the "vague" clinical history as well as the asymmetric and absurd appearance of the lesional skin, sev- eral nonrelated nail diseases had to be included in the differential diagnosis, such as acute contact dermatitis, acrodermatitis of Hallopeau, erosive/ulcerative lichen planus, SCC, and metastatic lesion.

Apart from the lack of specificity and the huge variability in nail picking manifestations, most patients tend to deny their debilitating compulsion, which further hinders undeniable diagnosis. Fortuitously, the launch of dermoscopy has dramatically expanded the diagnostic armamentarium of skin experts in recent years. Within this setting, Maddy and Tosti [9] accumulated their experience with dermoscopic features of onychotillomania in 2018, demonstrating that anonychia along with multiple obliquely arranged nail bed hemorrhages, nail bed gray pigmentation, and wavy lines are typical findings of the disorder not observed in other nail diseases. Scales and crusts usually embroider the above-described picture. Collectively, all these data accentuate that clinicopathologic correlation, incorporating dermoscopic images, is often warranted or even mandatory to secure a precise diagnosis $[3,9]$.

The ascertainment of the correct diagnosis is crucial for those sufferers, given the fact that onychotillomania may be an indicator of underlying psychopathology. That is to say, it seems to exhibit a high tendency to emerge over the course of multiple neuropsychiatric illnesses, including obsessive-compulsive disorder [1], depression [4, $5,7]$, as well as delusional disorders [1]. Hence, it could be a hint to unveil concurrent psychiatric abnormalities and vice versa. In other words, nail behavioral disorders may facilitate recognition of previously misdiagnosed mental deviations, as happened in the above-mentioned case. Conversely, among the mentally ill, thorough examination could reveal comorbid psychodermatological problems that may induce not only pain or discomfort, but also social or occupational dysfunction in the long run [4]. The self-limited course of our patient's lesion after placing the barrier between the mouth and fingers aroused suspicion about disturbed mental health, while the ensuing relapse after removing the occlusive bandage confirmed the consideration.

On top of that, Rieder and Tosti [2], in a review article in 2016, indicated that individuals often present with more than one behavioral disorder suggesting that there is some connection among various BFRBDs, including nail biting (onychophagia), habit-tic deformity, skin picking (dermatillomania), hair pulling (trichotillomania), and cheek biting. However, onychotillomania can also occur as a separate autonomous entity without coexisting mental illnesses, namely as a sole psychiatric 
problem [5]. Therefore, bearing in mind that this demeanor straddles the domains of both dermatology and psychiatry, it is imperative that dermatologists be vigilant about possible psychiatric comorbidity and have a low threshold to propose a mental health referral. In a high level of suspicion, thereby, psychiatric evaluation should be considered an integral part of the therapeutic regime [9], as the picking behavior pattern usually regresses with correction of the comorbid psychiatric condition, if any [1].

Despite the scarcity of data regarding the efficacy of treatment strategies, unpleasant-flavored topical agents and nail lacquers, physical barrier methods, cognitive-behavioral therapy, and psychopharmacological interventions have shown varying, yet encouraging therapeutic benefits $[1,2,10,11]$. One of the most commonly used practice is occlusion of the nails from trauma by placing a barrier between the mouth and fingers, e.g., occlusive dressings such as gloves, Unna boot, bandages, and wrappings with adhesive tape $[3,5,10]$. Our approach was to apply daily an occlusive bandage wrapping the fingernail and refer the patient to a psychiatrist for consultation and psychoactive medication after uncovering the concomitant psychopathology.
In conclusion, this case emphasizes the chameleonlike diversity in clinical presentations of onychotillomania, supporting the fact that compulsive nail picking might often be a stumbling block to differential diagnostic procedure. In addition, the reports of coexisting psychiatric disturbance underline the notion that in managing psychocutaneous disorders, a dual approach, addressing both dermatological and psychological aspects, is essential when encountering patients with suspected onychotillomania. However, more extensive research is clearly required so as to further elucidate etiological underpinnings and clinical characteristics of onychotillomania and determine the most efficient means to control and cease this behavioral disorder.

\section{Statement of Ethics}

The IRB of ATTIKON Hospital has approved the study and subjects have given their consent to publish photos and details of the case.

\section{Disclosure Statement}

All authors have no conflict of interest to disclose.

\section{References}

1 Singal A, Daulatabad D: Nail tic disorders: Manifestations, pathogenesis and management. Indian J Dermatol Venereol Leprol 2017;83:19-26.

2 Rieder EA, Tosti A: Onychotillomania: an underrecognized disorder. J Am Acad Dermatol 2016;75:1245-1250.

3 Reese JM, Hudacek KD, Rubin AI: Onychotillomania: clinicopathologic correlations. J Cutan Pathol 2013;40:419-423.

4 Bhardwaj A, Agarwal S, Koolwal A, Bhardwaj C, Sharma R: Onychotillomania as manifestation for underlying depressive disorder. Indian J Psychiatry 2016;58:98-99.
5 Grzesiak M, Pacan P, Reich A, Szepietowski JC: Onychotillomania in the course of depression: a case report. Acta Derm Venereol 2014; 94:745-746

6 Snorrason I, Woods DW: Nail picking disorder (onychotillomania): a case report. J Anxiety Disord 2014;28:211-214.

7 Lin YC, Lin YC: Onychotillomania, major depressive disorder and suicide. Clin Exp Dermatol 2006;31:597-599.

8 Keuthen NJ, Deckersbach T, Wilhelm S, Hale E, Fraim C, Baer L, O'Sullivan RL, Jenike MA: Repetitive skin-picking in a student population and comparison with a sample of self-injurious skin-pickers. Psychosomatics 2000; 41:210-215.
9 Maddy AJ, Tosti A: Dermoscopic features of onychotillomania: a study of 36 cases. J Am Acad Dermatol 2018, DOI: 10.1016/j. jaad.2018.04.015.

10 Halteh P, Scher RK, Lipner SR: Onychotillomania: diagnosis and management. Am J Clin Dermatol 2017; 18:763-770.

11 Magid M, Mennella C, Kuhn H, StamuO’Brien C, Kroumpouzos G: Onychophagia and onychotillomania can be effectively managed. J Am Acad Dermatol 2017;77:e143e144. 